\title{
Gated Geiger mode avalanche photodiode pixels with integrated readout electronics for low noise photon detection
}

\author{
E. Vilella ${ }^{\mathrm{a}, *}$, A. Comerma ${ }^{\mathrm{b}}$, O. Alonso ${ }^{\mathrm{a}}$, D. Gascon ${ }^{\mathrm{b}}$, A. Diéguez ${ }^{\mathrm{a}}$ \\ ${ }^{a}$ Department of Electronics, University of Barcelona (UB) Martí i Franquès 1, 08028 Barcelona, Spain \\ ${ }^{\mathrm{b}}$ Department of Structure and Constituents of Matter, University of Barcelona (UB) Martí $i$ Franquès 1, 08028 Barcelona, Spain
}

\section{A R T I C L E I N F O}

Keywords:

Geiger-mode avalanche photodiode Afterpulsing

CMOS

Dark count

Gated operation

Low light conditions

Low overvoltage

Low noise.

\begin{abstract}
A B S T R A C T
Avalanche photodiodes operated in the Geiger mode offer a high intrinsic gain as well as an excellent timing accuracy. These qualities make the sensor specially suitable for those applications where detectors with high sensitivity and low timing uncertainty are required. Moreover, they are compatible with standard CMOS technologies, allowing sensor and front-end electronics integration within the pixel cell. However, the sensor suffers from high levels of intrinsic noise, which may lead to erroneous results and limit the range of detectable signals. They also increase the amount of data that has to be stored. In this work, we present a pixel based on a Geiger-mode avalanche photodiode operated in the gated mode to reduce the probability to detect noise counts interfering with photon arrival events. The readout circuit is based on a two grounds scheme to enable low reverse bias overvoltages and consequently lessen the dark count rate. Experimental characterization of the fabricated pixel with the HV-AMS $0.35 \mu \mathrm{m}$ standard technology is also presented in this article.
\end{abstract}

(c) 2011 Elsevier B.V. All rights reserved.

\section{Introduction}

A great deal of emerging applications that require high sensitivity and low timing uncertainty photon detectors has appeared in recent years. These applications include 3-D imaging for medical purposes [1] or biological testing [2], time-of-flight ranging [3] and high energy physics experiments [4], to name a few. Sensor technologies available in the state-of-the-art, such as charge coupled devices (CCDs) [5], monolithic active pixel sensors (MAPS) [6], silicon photomultipliers (SiPMs) [7] and depleted field effect transistors (DEPFETs) [8], are generally not able to supply the desired accuracy and speed while keeping fabrication costs at reasonable levels.

The efficiency of Geiger-mode avalanche photodiodes (GAPDs) in single photon detection is well above the other options and has already been extensively reported in the literature [9]. GAPDs are basically $\mathrm{p}-\mathrm{n}$ junctions reverse biased above the breakdown voltage $\left(V_{\mathrm{BD}}\right)$. When a photon is absorbed by the depletion region, it may trigger a macroscopic self-sustained current pulse that can be easily detected by the readout electronics. However, due to the high intrinsinc gain of the sensor, thermal and tunneling generated carriers within the $\mathrm{p}-\mathrm{n}$ junction can also trigger avalanche pulses, which are indistinguishable from real photon-triggered pulses. The frequency of generation of these spurious pulses,

\footnotetext{
* Corresponding author. Tel.: +3493 40391 57; fax: +3493402 1148 .

E-mail address: evilella@el.ub.es (E. Vilella).
}

known as dark count rate (DCR), depends on the doping profile, the quality of the technology, the reverse bias overvoltage $\left(V_{\mathrm{OV}}\right)$ and the temperature. Moreover, charge carriers that were trapped during an avalanche flow also generate false pulses when they are released some time later and re-trigger the GAPD. The afterpulsing probability is a function of the trap density, the number of carriers generated during an avalanche and the lifetime of these carriers. The false pulses induce a high level of intrinsic noise that degrades the performance of the sensor. In addition, they also increase the amount of data that has to be stored for the subsequent processing.

Detectors based on GAPD sensors obtain information about the impinging radiation by counting the pulses generated during a certain period of time. However, noise pulses can lead to erroneous results in yes/no applications such as particle physics experiments. In addition, noise pulses also limit the range of detectable signals in light intensity measurements. Solutions commonly adopted to reduce the noise in GAPD detectors regard dedicated technologies with lower doping profiles [10], cooling methods [1] or advanced front-end circuits that minimize the avalanche charge flow [11]. However, none of the presented techniques is completely satisfactory due to high costs of fabrication, reduced applicability or efficiency in reducing the afterpulsing only. In this work, we present a GAPD pixel detector fabricated with the $0.35 \mu \mathrm{m}$ HV-AMS standard technology (h35b4), where the sensor and the front-end electronics have been monolithically integrated in the same single die, that makes use of two strategies to reduce noise pulses. On the one hand, the 
GAPD is operated in a gated mode [12] to enable the sensor only for short periods of time that are synchronized with the expected signal arrival. As a result, the afterpulsing probability is completely eliminated and the probability to detect dark counts interfering with real events (i.e., the dark count probability or DCP) is dramatically reduced. On the other hand, the pixel includes a readout circuit that can function with low $V_{\mathrm{OV}}$ to reduce the DCR. As it will be demonstrated, the combination of both methods allows to deeply reduce noise figures and widely improve the performance of the sensor.

\section{Pixel design and operation}

The schematic diagram of the proposed GAPD pixel is shown in Fig. 1. It is comprised of a rectangular GAPD with a sensitive area of $20 \mu \mathrm{m} \times 100 \mu \mathrm{m}$ and a suitable front-end circuit to run the gated operation with low $V_{\mathrm{OV}}$. The photodiode is implemented by means of a $\mathrm{p}^{+} /$deep $\mathrm{n}$-tub junction, which is surrounded by a p-tub implantation set to prevent premature edge breakdown. The breakdown voltage of the junction is $18.9 \mathrm{~V}$ and a $V_{\mathrm{DD}}$ of $3.3 \mathrm{~V}$ is applied to power the front-end circuit.

In contrast with the free-running mode of operation, where the sensor is always reverse biased above $V_{\mathrm{BD}}$ at a fixed voltage, in the gated operation the reverse bias voltage swings from above to below $V_{\mathrm{BD}}$ to consecutively enable and disable the photodiode. In this mode of operation, the sensor is kept active only for short periods of time that are synchronized with the expected signal arrival, thus improving its performance without any losses of information. The gating pulse is usually applied through AC coupling [13] or a high frequency sinousoidal voltage [14]. However, the former imposes a limitation on the repetition rate due to the coupling capacitor recharge time constant and the latter keeps variable the reverse bias of the sensor during gating periods. In the GAPD pixel proposed in this work, the gated operation is controlled by means of two external signals implemented through MOS transistors. When turned on by the RST signal, transistor $M_{N o}$ quickly recharges the sensor and the gated 'on' period is started. The RST pulse has to be as short as possible in order to minimize the presence of low resistive paths from the sensing node $\left(V_{S}\right)$ to ground. On the contrary, when the INH signal switches on transistor $M_{P 0}$, the polarization of the sensor is reduced below $V_{\mathrm{BD}}$, defining this way the gated 'off period. Transistor $M_{R}$ was included to allow different recharge times by means of an external adjustable $V_{\text {bias, }}$, but it is not used in the gated operation.
When an avalanche is triggered, the self-sustained current that flows through the junction charges the parasitic capacitance of the sensing node until its voltage has raised up to $V_{\mathrm{OV}}$. At this point, the polarization of the sensor has dropped down to $V_{\mathrm{BD}}$ and the avalanche is quenched. Node $V_{S}$ is connected to the readout electronics, which is based on a CMOS inverter $\left(M_{P 1}, M_{N 1}\right)$ that detects and digitizes avalanche voltages. Low $V_{\mathrm{OV}}$ are not allowed in this technology given that the threshold voltage of nMOS transistors is set at $0.5 \mathrm{~V}$. In order to overcome this drawback, the sensor and the readout circuit have different ground nodes (GND and $V_{\mathrm{SS}}$ ). By raising GND with regard to $V_{\mathrm{SS}}$, low avalanche voltages can be easily detected by the CMOS inverter, whose threshold voltage is set at $V_{\mathrm{DD}} / 2$.

The output of the readout is fed in a dynamic latch $\left(M_{N 2}, M_{P 2}\right.$ and $M_{N 3}$ ) which has been included to make possible in-pixel storage. The sampling periods of the dynamic latch are synchronized with the active periods of the sensor by means of the external signal CLK1. This signal switches on pass gate $M_{N 2}$ at the beginning of the gated 'on' period and switches it off a few nanoseconds before the gated 'off period is started to avoid storing a false ' 1 '. The interval of time in which the dynamic latch is sampling the inverter output is called period of observation $\left(t_{\mathrm{obs}}\right)$. The value of $V_{S}$ at the end of $t_{\mathrm{obs}}$ is stored during the gated 'off period. The pixel is read when pass gate $M_{N 4}$ is turned on by the external signal CLK2. The waveforms to control the GAPD in the gated operation are depicted in Fig. 2.

\section{Experimental set-up and measured results}

A microphotograph of the prototype fabricated with the $0.35 \mu \mathrm{m}$ HV-AMS standard technology (h35b4) can be seen in Fig. 3 together with the experimental set-up. To demonstrate the efficiency of the proposed methods to reduce the noise in GAPD detectors, the response of the pixel in darkness and also to light was tested at room temperature. The chip was mounted on a printed circuit board and powered with an Agilent E3631A voltage source. An Altera Stratix II FPGA-based control board was used to generate the fast logic control signals (RST, INH, CLK1 and CLK2) and also to count off-chip the number of pulses generated by the sensor. The optical response of the pixel to a variable intensity of a $880 \mathrm{~nm}$ light was studied by placing an infrared led over the sensor. The light emitter was powered using an HP 3245 A universal source and the current flowing through it was measured by means of an HP 3458 A multimeter. The chip, together with the FPGA and the infrared led, was placed inside a

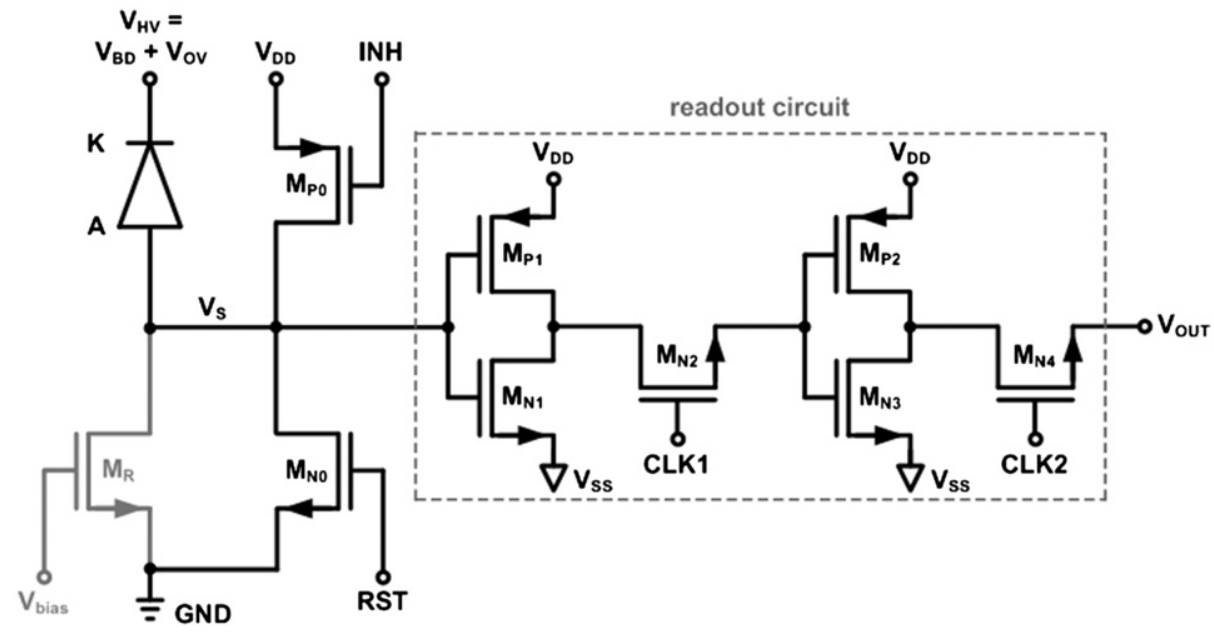

Fig. 1. Schematic diagram of the proposed pixel detector. 


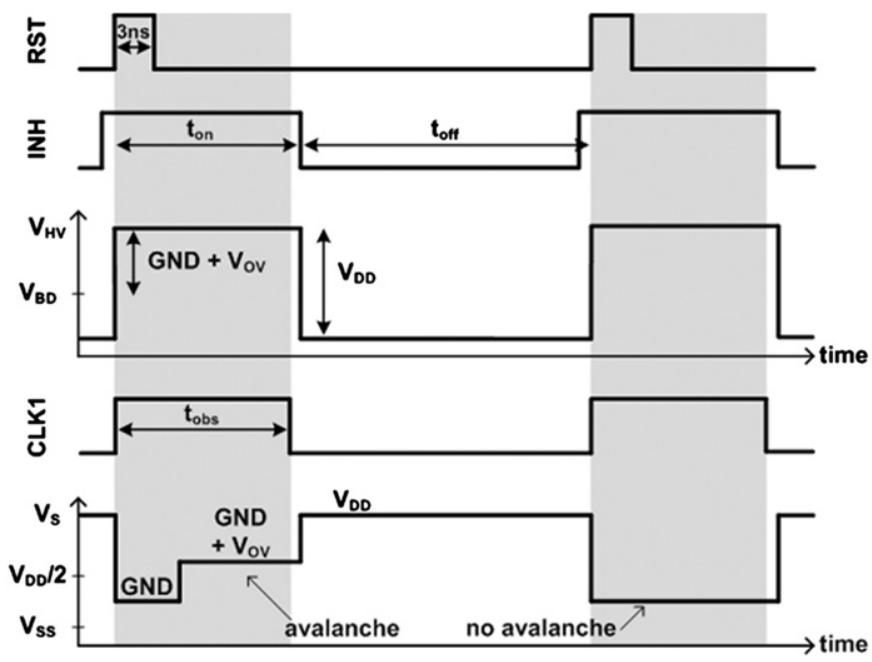

Fig. 2. Waveforms for the gated operation and dynamic readout.

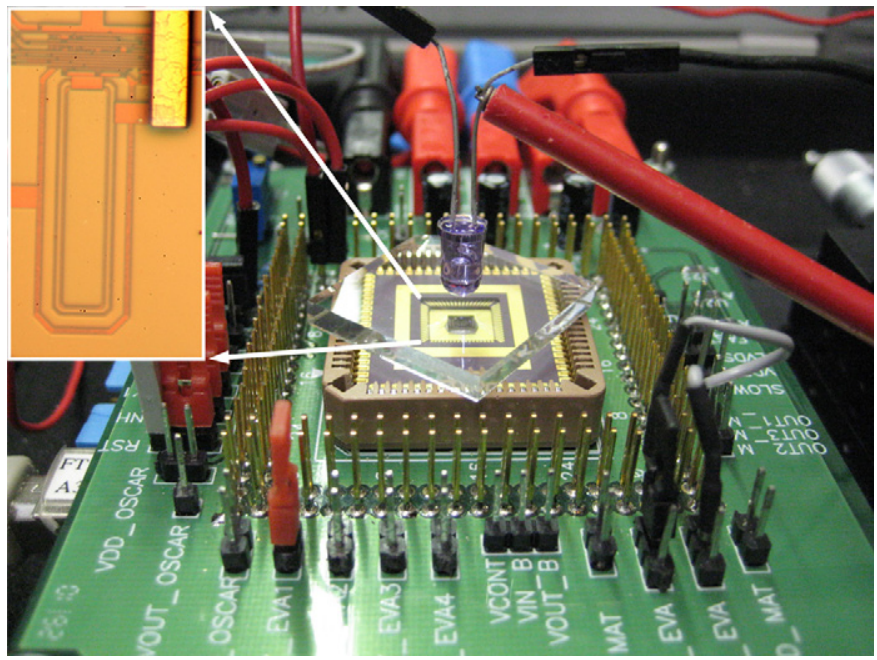

Fig. 3. Set-up for the characterization of the optical response and microphotograph of the pixel.

metallic box to protect the circuit from electromagnetic inteferences and uncontrolled light sources. The measurements were done with an adjustable integration time that depends on the period of observation of the sensor and also on the number of times that the observation is repeated $\left(n_{\mathrm{rep}}\right)$. Different $t_{\mathrm{obs}}$ that range from $10 \mathrm{~ns}$ to $2560 \mathrm{~ns}$ were analyzed for $10^{5}$ repetitions. The behavior of the pixel was characterized for three different $V_{\mathrm{OV}}$ of $0.2 \mathrm{~V}, 0.5 \mathrm{~V}$ and $0.8 \mathrm{~V}$.

Firstly, the afterpulsing probability was tested by leaving different gated 'off periods for a fixed $t_{\mathrm{obs}}$ of $10 \mathrm{~ns}$. It was observed that gated 'off' periods of $300 \mathrm{~ns}$ are enough to eliminate afterpulses at a $V_{\mathrm{OV}}$ of $1.0 \mathrm{~V}$, which shows that the lifetime of the trapping levels is shorter than this time. Secondly, the DCR of the sensor was characterized for different $t_{\mathrm{obs}}$ with a $t_{\mathrm{off}}$ of $300 \mathrm{~ns}$. This parameter is obtained by dividing the number of generated false counts by the product of $t_{\mathrm{obs}}$ and $n_{\text {rep. }}$. As shown in Fig. 4, the DCR is constant despite the value of $t_{\mathrm{obs}}$ due to its random nature. This implies that the number of observed dark counts is reduced linearly for shorter $t_{\mathrm{obs}}$, as it can be deducted from dark counts $=D C R t_{\text {obs. }}$. For example, given that the DCR is $43 \mathrm{kHz}$ for a $V_{\mathrm{OV}}$ of $0.2 \mathrm{~V}$, with a $t_{\mathrm{obs}}$ of $10 \mathrm{~ns}$ only one dark count will be seen each 2325 repetitions of the measurement. For a $t_{\mathrm{obs}}$ of $20 \mathrm{~ns}$, this rate increases up to 1162 repetitions, and so on for longer $t_{\mathrm{obs}}$.

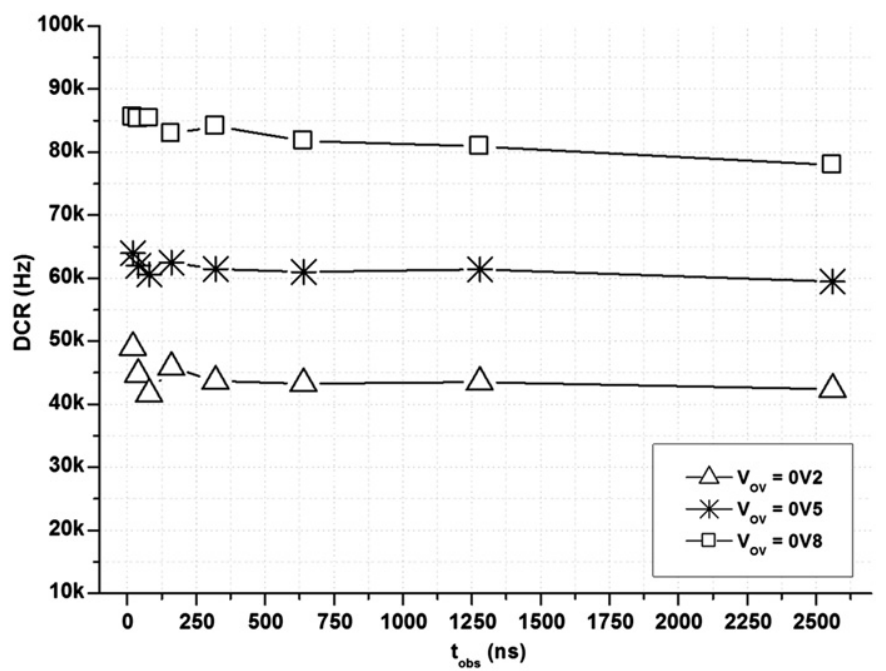

Fig. 4. DCR of the proposed pixel for different $V_{\mathrm{OV}}$.

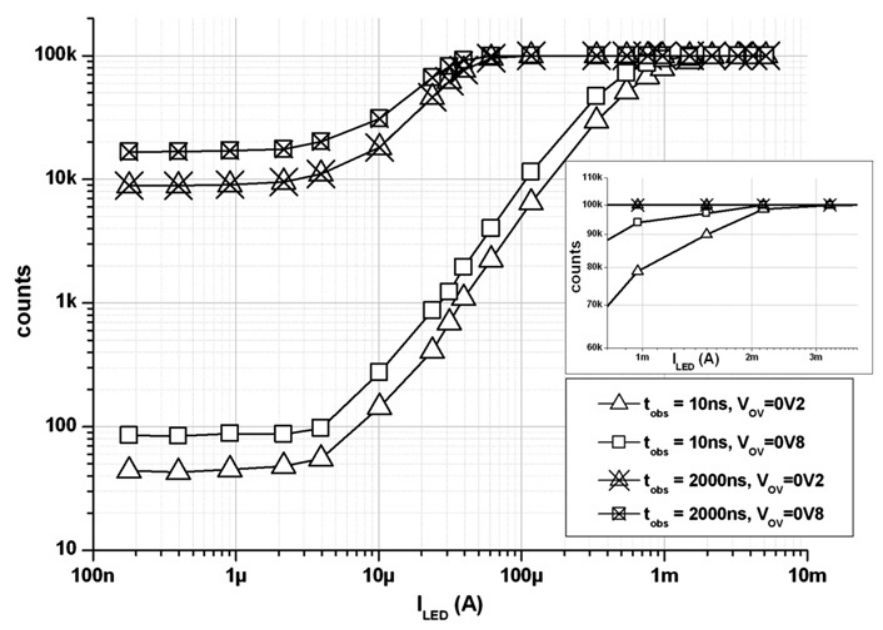

Fig. 5. Response of the GAPD pixel to a light intensity of $880 \mathrm{~nm}$ for different $t_{\mathrm{obs}}$ and $V_{\mathrm{OV}}$.

As a consequence, in those applications where the signal to be detected is present only in a well defined interval after a triggering signal, as it happens in the vast majority of current applications, the gated operation with discrete $t_{\mathrm{obs}}$ in the nanosecond range allows to dramatically reduce the probability to detect dark counts without missing any photon count. Moreover, it has also been detected that low $V_{\mathrm{OV}}$ further reduce the DCR, as expected.

The optical response of the pixel to a variable light was tested (see Fig. 5). The experiment was done for two different $t_{\mathrm{obs}}$ of $10 \mathrm{~ns}$ and $2000 \mathrm{~ns}$ at two different $V_{\mathrm{OV}}$ of $0.2 \mathrm{~V}$ and $0.8 \mathrm{~V}$ with a counter of a maximum capacity of $10^{5}$ counts. For each operation point (fixed $t_{\mathrm{obs}}$ and $V_{\mathrm{OV}}$ ), the sensor was illuminated with a certain light intensity and its response observed for $10^{5}$ times. Several light intensities were measured until the generated counts caused counter saturation. At low light intensities the detected counts are dark counts only and no signal counts are appreciated. The threshold intensity corresponds to the minimum light intensity from which signal counts above the noise floor are obtained. As shown in Fig. 5, this value is set at $3.95 \mu \mathrm{A}$, regardless of $t_{\mathrm{obs}}$ and $V_{\mathrm{OV}}$. This demonstrates that our techniques are not limiting the sensor capabilities. Given that the number of detected dark counts is lower for a shorter $t_{\mathrm{obs}}$, a wider range of 
light intensities can be measured with the 10 ns gated case until counter saturation. On the contrary, if the detected dark counts are higher, as it happens in the 2000 ns gated case, the counter reaches saturation for a lower value of the incident light intensity and the range of measurable signals becomes narrow. Therefore, it is observed that reduced noise floors due to shorter $t_{\text {obs }}$ extend the range of sensitivity of light measurements in triggered applications with a fixed counter (as it happens in standard systems). The dynamic range (DR) of the detector, defined here as the number of bits necessary to cover the range of values given by the ratio of the saturation $\left(I_{\text {sat }}\right)$ and the threshold $\left(I_{\mathrm{th}}\right)$ intensities and expressed as $\mathrm{DR}=\log _{2}\left(I_{\mathrm{sat}} / I_{\mathrm{th}}\right)$, is increased from 6 to 11 bits by reducing the $t_{\mathrm{obs}}$ from $2000 \mathrm{~ns}$ to $10 \mathrm{~ns}$. Apart from that, it is also known that a higher photon detection efficiency (PDE) can be obtained with a higher $V_{\mathrm{OV}}$. As it can be observed in Fig. 5, a higher number of counts is obtained with a higher $V_{\mathrm{OV}}$ for the same $t_{\text {obs. }}$. However, an increased number of counts due to a higher $V_{\mathrm{OV}}$ may cause counter saturation for a lower light intensity and reduce the DR in a system with a fixed counter. It is concluded that the gated operation with short $t_{\mathrm{obs}}$ and low $V_{\mathrm{OV}}$ allows an extension of the DR.

\section{Conclusion}

A pixel detector based on GAPDs operated in the gated mode has been fabricated with the $0.35 \mu \mathrm{m}$ HV-AMS standard technology. The proposed mode of operation allows to synchronize the gated 'on' periods of the sensor with the expected signal arrival. It has been demonstrated that short gated 'on' periods in the nanosecond range, together with suitable readout electronics that allow low reverse bias overvoltages, completely eliminate afterpulses and dramatically reduce the number of observed dark counts. In addition, a dynamic range of 11 bits is achieved with a gated 'on' period of $10 \mathrm{~ns}$ with these detectors.

\section{Acknowledgments}

This work has been partially supported by the National Program for Particle Physics through the projects "Desarrollo de nuevas tecnologías en aceleradores y detectores para los futuros colisionadores de Física de Partículas", coded FPA2008-05979C04-02, and "Desarrollo de nuevos detectores para los Futuros colisionadores en Física de Partículas”, coded FPA2010-21549C04-01.

\section{References}

[1] S. McCallum, P. Clowes, A. Welch, Physics in Medicine and Biology 50 (2005) 4187.

[2] D. Stoppa, D. Mosconi, L. Pancheri, L. Gonzo, IEEE Sensors Journal 9 (2009) 1084.

[3] C. Niclass, A. Rochas, P.A. Besse, E. Charbon, IEEE Journal on Selected Topics in Quantum Electronics 10 (2004) 796

[4] A. Arbat, J. Trenado, D. Gascon, A. Vilà, A. Comerma, L. Garrido, A. Diéguez, Proc. SPIE Opt, Photonics 7780 (2010) 77801G.

[5] K.D. Stefanov, Nucl. Instrum., Methods Phys. Res., Sect. A 549 (2005) 93.

[6] G. Deptuch, G. Claus, C. Colledani, M. Deveaux, A. Gay, W. Dulinski, W. Gornushkin, C. Hu-Guo, M. Winter, Nuclear Instruments and Methods in Physics Research Section A: Accelerators, Spectrometers, Detectors and Associated Equipment 511 (2003) 240.

[7] T. Frach, G. Prescher, C. Degenhardt, R. Gruyter, A. Schmitz, R. Ballizany, IEEE Nucl. Sci. Symp. Conf. Record (2009) 1959.

[8] J.J. Velthuis, R. Kohrs, M. Mathes, A. Raspereza, L. Reuen, L. Andricek, M. Koch, Z. Dolezal, P. Fischer, A. Frey, F. Giesen, P. Kodys, C. Kreidl, H. Krüger, P. Lodomez, G. Lutz, H.G. Moser, R.H. Richter, C. Sandow, D. Scheirich, E. von Törne, M. Trimpl, Q. Wei, N. Wermes, Nucl. Instrum. Methods Phys. Res., Sect. A 579 (2007) 685.

[9] F. Zappa, S. Tisa, A. Tosi, S. Cova, Sensors Actuators, A 140 (2007) 103.

[10] M. Gersbach, J. Richardson, E. Mazaleyrat, S. Hardillier, C. Niclass, R. Henderson, L. Grant, E. Charbon, Solid State Electronics 53 (2009) 803.

[11] S. Tisa, F. Guerrieri, F. Zappa, Optic Express 16 (2008) 2232.

[12] A. Lacaita, F. Zappa, S. Cova, P. Lovati, Applied Optics (1996) 2986.

[13] O. Thomas, Z.L. Yuan, J.F. Dynes, A.W. Sharpe, A.J. Shields, Applied Physics Letters 97 (2010) 031102.

[14] N. Namekata, S. Sasamori, S. Inoue, Eur. Conf. Opt. Commun 24-28 (2006) 1. 